Original Research Paper

\title{
Carica papaya Fruit Attenuates Apoptotic Degeneration After Permanent Cerebral Ischemia Rat
}

\author{
${ }^{1,2}$ Pratchaya Kaewkaen \\ ${ }^{I}$ College of Research Methodology and Cognitive Science, Burapha University, Thailand 20131, Thailand \\ ${ }^{2}$ Animal Cognitive Neuroscience Laboratory (ACoN), Burapha University, Thailand 20131, Thailand
}

Article history
Received: 21-07-2018
Revised: 25-10-2018
Accepted: 19-11-2018
Email: pratchaya@buu.ac.th

\begin{abstract}
Apoptotic neurodegeneration is a phenomenon following cerebral ischemia. Both the increased caspase-3 and decreased Bcl-2 expression are reported to play crucial role on apoptosis in neuron. In addition, phytonutrient can successfully increase the endogenous antioxidant and lower the apoptotic responses. Therefore, anti-apoptotic effect of Carica papaya fruits was examined. To the best of our knowledge, no scientific effect was available. Therefore this study aimed to determine the effect of Carica papaya on neuron density and the densities of both caspase-3 and Bcl-2 immunopositive neuron in hippocampus. Adult male Wistar rats, weighing 280-320 g, were orally given Carica papaya fruits extract at doses of 50,150 and $450 \mathrm{mg} / \mathrm{kg}$ at a period of 14 days before and 14 days after the occlusion of right Middle Cerebral Artery (Rt.MCAO). The results showed that Carica papaya fruits at high doses used in this study significantly attenuated the decreased neuron density in hippocampus. In addition, the high doses of Carica papaya could decrease caspase-3 immunopositive neuron density but increased density of Bcl-2 immunopositive neurons in hippocampus. The decreased caspase- 3 immunopositive neuron density and the increased Bcl-2- immunopositive neurons might be partly responsible for neuroprotective effect of Carica papaya. Therefore, Carica papaya is the potential fruit to protect against apoptotic neurodegeneration following cerebral ischemia. However, further researches are essential to elucidate the possible mechanism.
\end{abstract}

Keywords: Carica papaya, Apoptosis, Neurodegeneration, Cerebral Ischemia

\section{Introduction}

Cerebrovascular disease or stroke is the sudden death of the brain tissue due to the lack of oxygen when the cerebral blood flow is impaired by the blockage or rupture of an artery to the brain. According to the Bulletin of the World Health Organization (Sacco et al., 2013), stroke is defined as "a syndrome of rapidly developing clinical signs of focal (or global) disturbance of cerebral function, with symptoms lasting $24 \mathrm{~h}$ or longer or leading to death, with no apparent cause other than of vascular origin." Of all strokes, $88 \%$ are ischemic, 9\% involve an intracerebral hemorrhage and $3 \%$ involve a subarachnoid hemorrhage. The most common type of stroke is atherothrombotic brain infarction, which accounts for approximately $61 \%$ of all strokes (excluding transient ischemic attacks). The second most common type of stroke is embolic stroke, at 22\% (Wang-Fischer, 2009). The ischemic stroke occur as arterial occlusion and thromboembolism. Under normal circumstance, the brain requires blood supply approximately $55 \mathrm{ml} / 100 \mathrm{~g} / \mathrm{min}$. It has been previously shown that the cellular metabolic abnormalities are observed when the cerebral blood flow decreased to 20$30 \mathrm{ml} / 100 \mathrm{~g} / \mathrm{min}$ (Markus, 2004). This condition is recognized as cerebral ischemia and can be induced by various causes including the thrombotic or embolic occlusion of a major cerebral artery particularly Middle Cerebral Artery (MCA) (Ren et al., 2012). When the cerebral blood flow insufficiency had occurred, brain infarctions appeared to depend on $\mathrm{n}$ the intensity and time duration of ischemia. It has been reported that 
when the brain area which blood flow level decreases below 10 to $25 \%$ or approximate $20 \mathrm{ml} / 100 \mathrm{~g} / \mathrm{min}$, the inadequate supply of oxygen and glucose occurs resulting in the rapid depletion of energy stores and finally giving rise to the degeneration of both neurons and glial (Mergenthaler et al., 2013). This area has been regarded as infarct core or the core zone area. In addition to the core zone, the degeneration also occurs in the area lying between the normal area and the core zone which has been known as penumbra area.

Presently, the most neuroprotective strategies target at the penumbra area in order to rescue and attenuate the neurodegeneration in this area (Liu et al., 2012). Traditionally, it has been believed that neurodegeneration after cerebral ischemia was considered to be exclusively necrotic in nature. However, accumulative lines of evidence during the past decade have revealed that after a stroke, many neurons in the ischemic penumbra will undergo apoptosis (Broughton et al., 2009). Cerebral ischemia induced by stroke has been reported to enhance the production of oxygen free radicals and other reactive oxygen species. which in turn serve as essential intermediate messenger molecules in cascade phenomenon of apoptosis (Slater et al., 1995). Reactive oxygen species can be scavenged through utilizing natural antioxidant compounds present in foods and medicinal plants. In addition, recent evidence shows that naturally occurring phenolic compounds can successfully increase the endogenous antioxidant and lower the apoptotic responses (Raza et al., 2011).

The papaya (Carica papaya Linn.) is an economically imperative crop of tropical and subtropical regions, is cultivated in over 50 different countries of the world. Numerous biologically active moieties are present in papaya. It has wide consumption owing. The pharmacological properties of Carica papaya can be used as a folk remedy for various disorders because it contains different kinds of immuno-stimulating agents and antioxidants (Aruoma et al., 2006; Mehdipour et al., 2006). It is rich in cysteine endopeptidases having glycyl endopeptidase, cysteine proteinases, serine proteinase inhibitor, glutaminyl cyclase caricain, class II chitinase, papain and chymopapain. Proteases extracted from papaya exhibit a broad specificity and thermo stability thus utilized in the meat industry for meat tenderization. Papaya proteases are of medicinal significance especially for gastroenterology, wound healing, anti-inflammatory, antitumoral, neurosurgery, ophthalmology, urology and phlebology properties (Pendzhiev, 2002; Seki et al., 2007). Even though there are some reports about nutritional and chemical contents in papaya fruits worldwide, there is still lack of the information about the carotenoid contents in the fruits of papaya varieties cultivated in Thailand. Carotenoids are hydrophobic terpenoidal compounds which promoted the protective effects against some types of cancer, aging-related molecular degeneration (Ma et al., 2012) and heart disease (Palace et al., 1999) while some clinical studies revealed that the carotenoid also play important roles in prevention of chronic diseases related to oxidative stress such as cancer, diabetes and cardiovascular disease. (Rojsanga et al., 2014). Based on these pieces of information, the anti-apoptotic effect of substance of diet which is rich in total carotenoid has been considered.

\section{Materials and Methods}

\section{Animals}

Adult male Wistar rats (280-320 g, 8 weeks old) were obtained from National Laboratory Animal Center, Salaya, Nakorn Pathom, Thailand and were housed in group of 4 per cage in standard polycarbonate cages at $22 \pm 2^{\circ} \mathrm{C}$ on $12: 12 \mathrm{~h}$ light-dark cycle. All animals were given access to food and water ad libitum. All the procedures carried out in this study were approved by the Institutional Animal Care and Use Committee.

\section{Sample Preparation}

Ripen Carica papaya fruit (Srisupa species) provided from an organics farm in Bang Lamung District, Chon Buri Province, Thailand. Carica papaya (the starting mass material was approximately $5 \mathrm{~kg}$ ) were washed and selected to provide a homogeneous group, based on date of harvest, color, size and freshness according to a visual analysis. Both the upper and lower ends of the fruits were rejected and then cut into longitudinal sections. Both seeds and mucilage were removed and thereafter the fruit with skin was homogenized in a blender for further analyses.

\section{Determination of Total Carotenoids and Chlorophylls $a$ and $b$}

Methanolic solutions of plant extracts of the appropriate concentration ( 1.0 to $4.0 \mathrm{mg} / \mathrm{ml}$ ) were analyzed in a He $\lambda$ ios $\alpha$ UV/VIS spectrophotometer at 470,653 and $666 \mathrm{~nm}$. The concentrations of carotenoids and chlorophylls $\alpha$ and $b$ were determined according to the equations reported by Lichtenthaler and Wellburn (1985) and Rainha et al. (2011) as follows:

- $\quad$ Total carotenoids $(\mathrm{mg} / \mathrm{L})=1000 \mathrm{Abs}_{470}-2.860 \mathrm{C}_{\mathrm{a}}$ $129.2 \mathrm{C}_{\mathrm{b}} / 245$

- Chlorophyll $\alpha(\mathrm{mg} / \mathrm{L})=15.65 \mathrm{Abs}_{666}-7.340 \mathrm{Abs}_{653}$

- Chlorophyll b (mg/L) = 27.05 $\mathrm{Abs}_{653}-11.21 \mathrm{Abs}_{666}$

\section{Experimental Protocol}

All rats were randomly divided into 4 groups following, Group 1: Vehicle treated group. (Distilled 
water) Group 2-4: Carica papaya fruits treated groups. Rats in these groups were administered Carica papaya fruits at various doses ranging from 50, 150 and 450 $\mathrm{mg} / \mathrm{kg}$ respectively via oral route.

All animals were treated with vehicle, or Carica papaya at a period of 14 days before and 14 days after right Middle Cerebral Artery Occlusion (MCAO). At the last day of experiment, the rat brains were removed to determine the neuronal density via Cresyl violet staining, Immunohistochemical staining of Bcl-2 and caspase-3 immunopositive neurons.

\section{The Surgical Procedure}

The Intra-luminal suture model was developed in the rat by Koizumi et al. (1996), modified by Longa et al. (1989) Permanent Middle Cerebral Artery Occlusion (pMCAO) was performed at the right middle cerebral artery (MCAO) using an intraluminal filament technique of Longa. In brief, rats were anesthetized by thiopental sodium at dose of $50 \mathrm{mg} / \mathrm{kg}$. The right common carotid artery and the right external carotid artery were exposed through a ventral midline neck incision and they were ligated proximally. A silicone coated nylon monofilament (4-0) suture (USS DGTM sutures; Tyco Healthcare group LP, Connecticut, USA) with its tip rounded was inserted through an arteriectomy in the common carotid artery just below the carotid bifurcation and then advanced into the internal carotid artery approximately $17-18 \mathrm{~mm}$ distal to the carotid bifurcation until a mild resistance was felt. Occlusion of the origins of the anterior cerebral artery, the middle cerebral artery and the posterior communicating artery was thereby achieved.

Then, the wound was sutured, the rats were returned to their cages with free access to food and water. The incision sites were infiltrated with $10 \%$ Povidone- Iodine Solution for anti-septic postoperative care.

Survival Neurons of Hippocampus (Cresyl Violet Staining for Nissl Substance)

Adjacent series of sections containing hippocampus of all treated groups were stained with $0.5 \%$ cresyl violet to aid in neuronal death density determination. The neuron density in various regions of hippocampus was observed under light microscope (Olympus light microscope model CX-21; made in Japan) at $40 \mathrm{X}$ magnification by blinded observer.

Immunohistochemical Staining of Bcl-2 and Caspase-3 Immunopositive Neurons

This part of study focusing on Immunohistochemical stain, A series of sections containing hippocampus were reacted in a mouse monoclonal antibodies directed against either Bcl-2 or Caspase-3 (Chemicon Internation, Inc., CA, USA) and a modification of a previously described protocol employing the DAKO Strept ABC Complex/HRP duet kit. In brief, the sections were eliminated endogenous peroxidase activity by $0.5 \% \quad \mathrm{H}_{2} \mathrm{O}_{2}$ in methanol. Sections were washed in running tap water and distilled water for 1 minute each, then rinsed in KPBS and KPBS-BT for 5 minutes per each process. Excess buffer was removed and then incubated for $30 \mathrm{~min}$ in a blocking solution composed of 5\% normal goat serum in KPBS-BT. Then, the sections were incubated in mouse primary antibody against Bcl-2 diluted 1: 400 in KPBS-BT at room temperature for $2 \mathrm{~h}$ and incubate at $4^{\circ} \mathrm{C}$ for $48 \mathrm{~h}$. The tissue was rinsed in KPBS-BT ( 2 washes $\times 7 \mathrm{~min}$ ), incubated for $1 \mathrm{~h}$ in biotinylated goat antimouse $\mathrm{IgG}$ antibody, rinsed in KPBS-BT ( 2 washes $\times 7 \mathrm{~min}$ ) and then incubated in Strept ABC Complex/HRP for $4 \mathrm{~h}$. The sections were rinsed in KPBS-BT $(1 \mathrm{~min})$ and KPBS $(2$ washes $\times 10$ min). Bcl-2 immunoreactivity was visualized using $0.025 \%$ 3, 3' Diaminobenzedine (DAB, Sigma) and $0.01 \% \mathrm{H} 2 \mathrm{O} 2$. for $24 \mathrm{~h}$. Finally, sections were rinsed in running tap water, air dried and cover-slipped using permount (Kaewkaen et al., 2012).

\section{Statistical Analysis}

Data were presented as mean \pm Standard Deviation (SD). The analysis was performed using one-way Analysis Of Variance (ANOVA), followed by LSD test. All statistical results were considered significant at $\mathrm{p}$-value $<0.05$.

\section{Results}

Table 1 show the spectrophotometrical measurement of total carotenoids based at the sample Abs at $470 \mathrm{~nm}$. The results revealed that the Carica papaya is the rich of carotenoid ingredient.

The effects of Carica papaya on neuron density and densities of both Caspase-3 and BCl-2 immunopositive neuron were shown in Fig. 1-3. It was found that rats subjected to high doses of Carica papaya used in this study significantly enhanced neuron density in hippocampus CA1, CA2, CA3 and Dentate gyrus (DG) ( $p$-value $<0.05$; compared to vehicle + MCAO treated group). Figure 4 whereas rats subjected to medium dose of Carica papaya showed the increased density of Bcl-2 immunopositive neurons in CA1 p-value $<0.05$, compared to vehicle $+\mathrm{MCAO}$ treated group).

Table 1: Total carotenoids of Carica papaya (Srisupa Species)

\begin{tabular}{lc} 
Sample & Total carotenoids $(\mathrm{mg} / \mathrm{l})$ \\
\hline *The values are mean \pm standard deviation $(\mathrm{n}=3)$
\end{tabular}




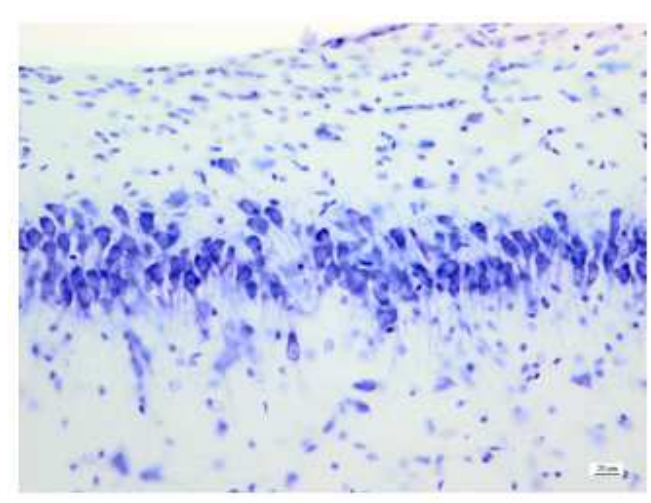

Vehicle group (distilled water) $+\mathrm{MCAO}$

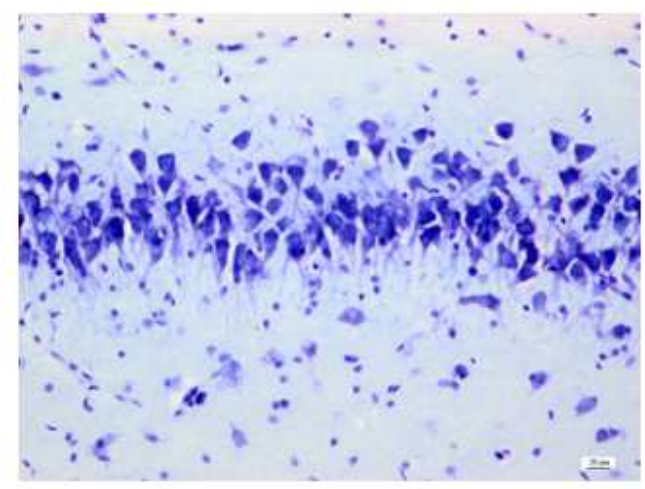

Carica papaya $150 \mathrm{mg} / \mathrm{kg}+\mathrm{MCAO}$

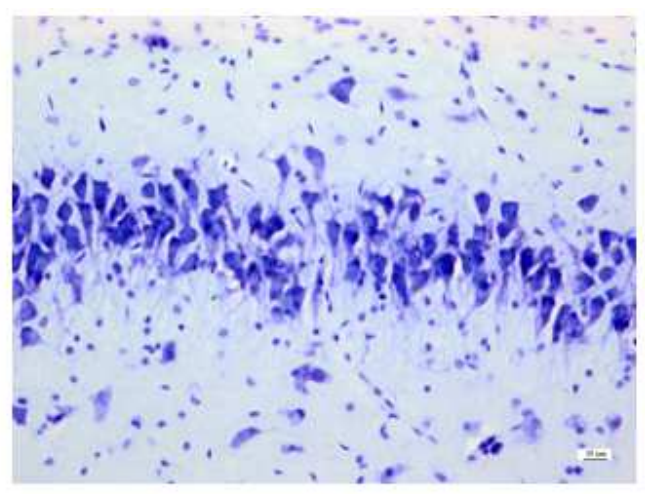

Carica papaya $50 \mathrm{mg} / \mathrm{kg}+\mathrm{MCAO}$

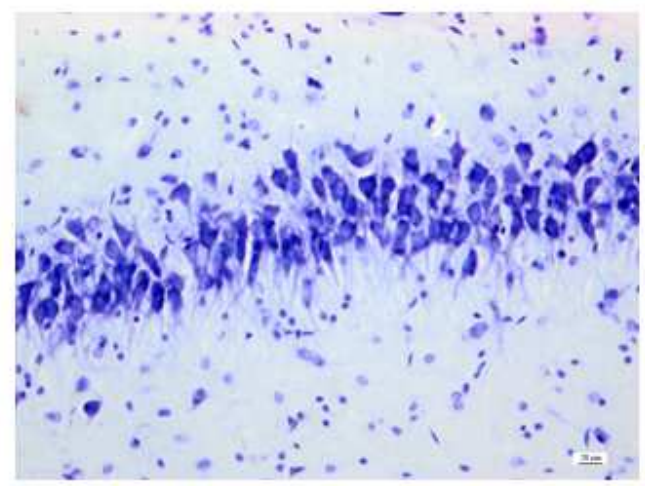

Carica papaya $450 \mathrm{mg} / \mathrm{kg}+\mathrm{MCAO}$

Fig. 1: The survival neuron stain in hippocampus CA3

200.00

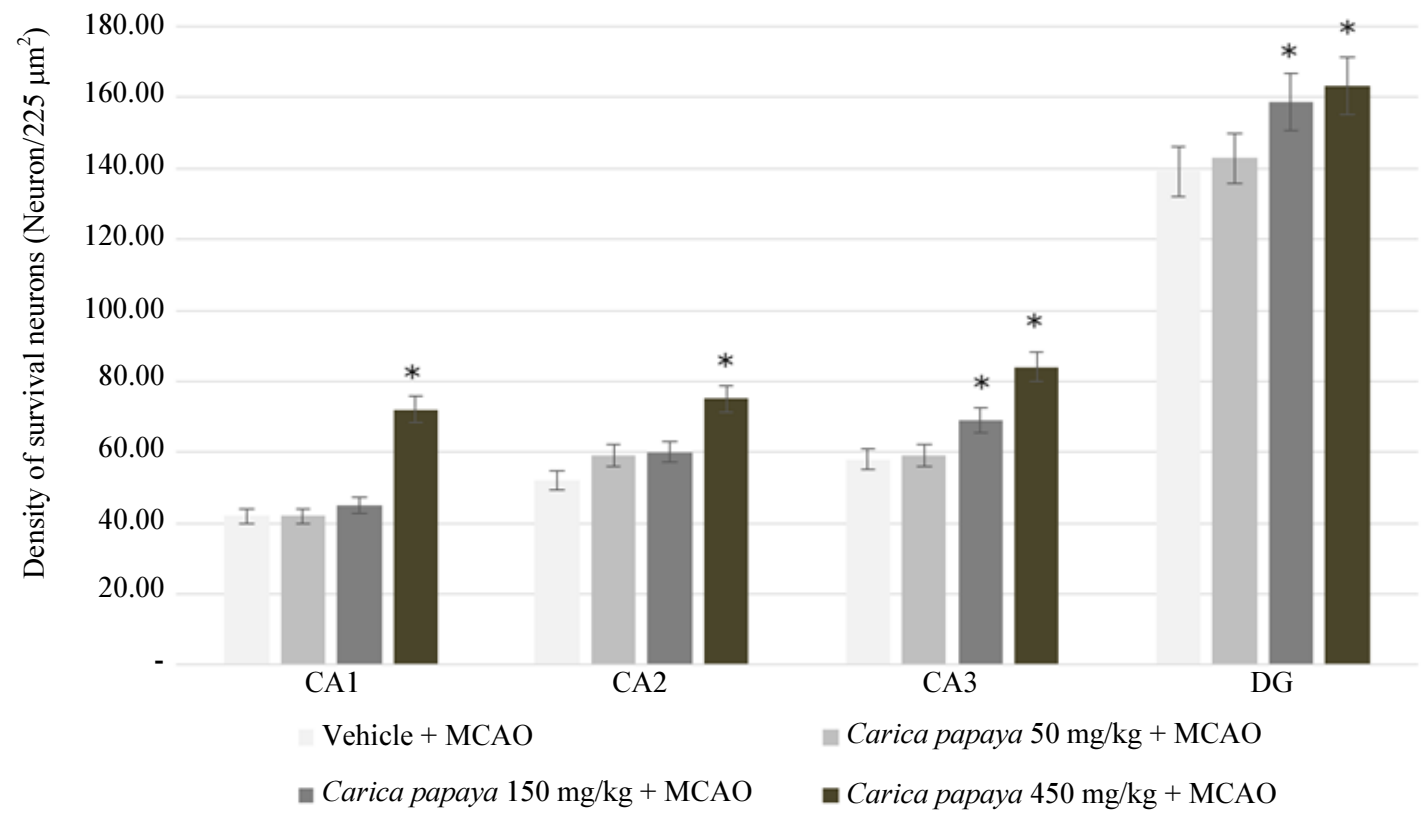

Fig. 2: The effect of Carica papaya on survival neuron in hippocampus 


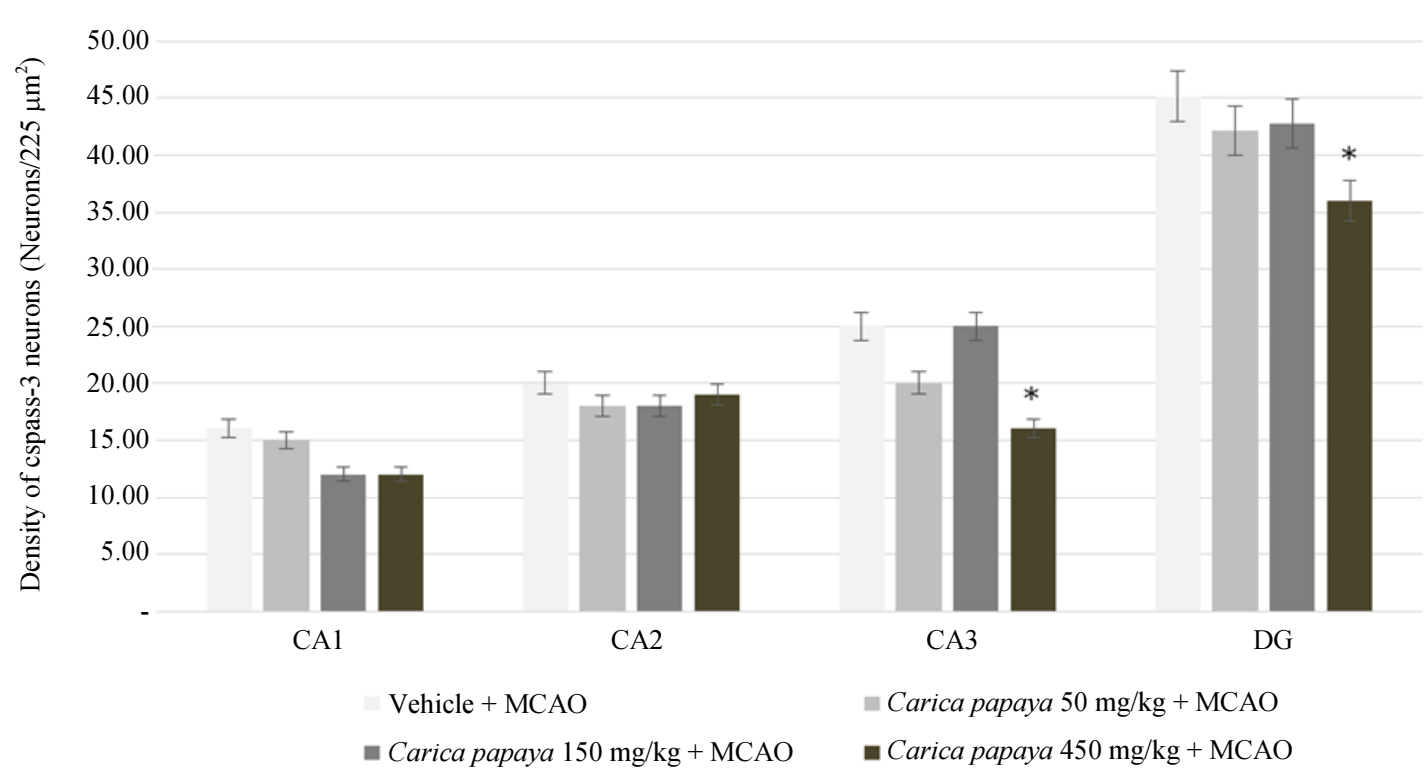

Fig. 3: The effect of Carica papaya on caspase-3 immunopositive neurons in hippocampus

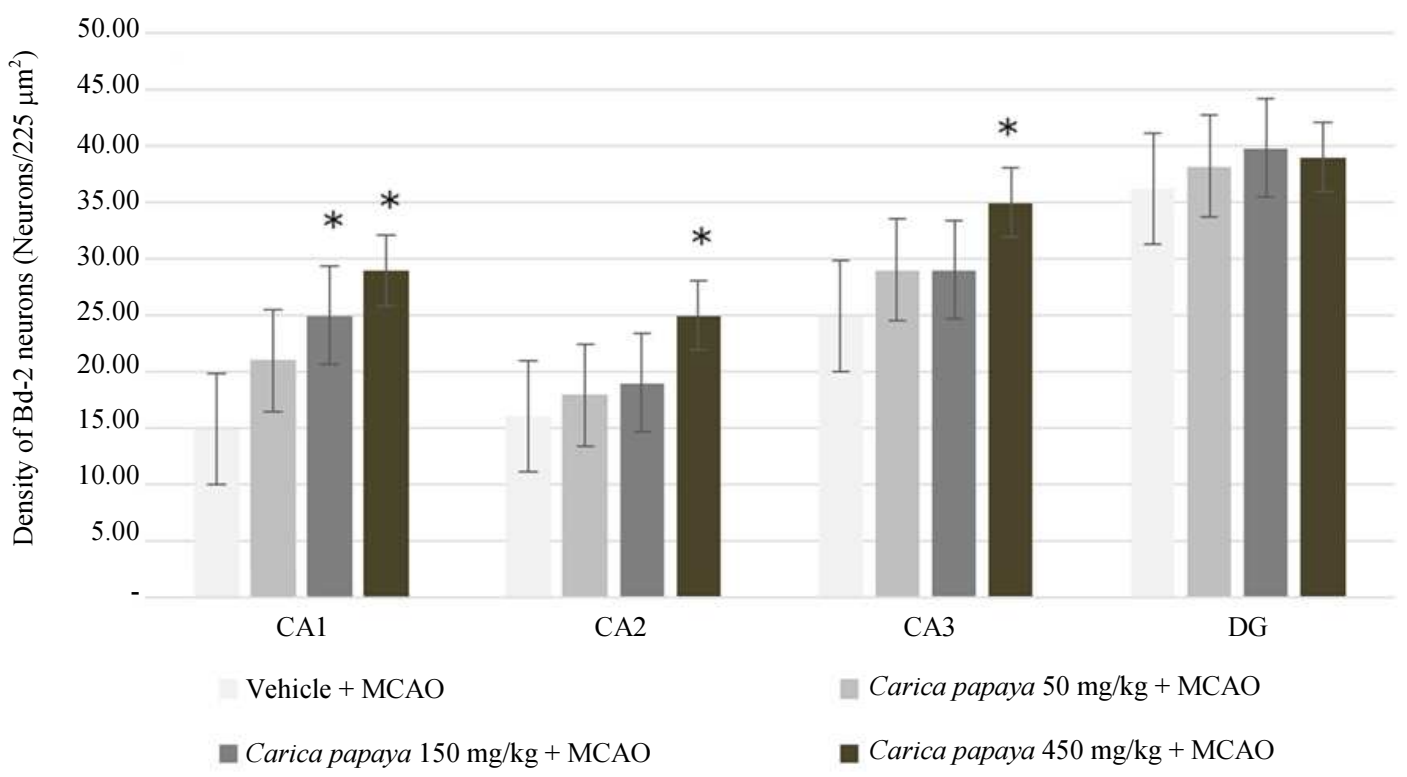

Fig. 4: The effect of Carica papaya on Bcl-2 immunopositive neurons in hippocampus

\section{Discussion}

In the present study, we demonstrated that Carica papaya could enhance the densities of neuron in hippocampus. In addition, it enhanced density of Bcl-2immunopositive neurons in hippocampus CA1 and CA3 and decrease caspase- 3 immunopositive neurons in hippocampus CA2 and CA3.

Apoptotic cell death, or programmed cell death plays a crucial role on neurodegeneration following cerebral ischemia (Chen et al., 1998). Recent evidence showed that apoptosis-related cell death is linked to caspase activation. Kim et al. (2000) especially caspase-3, a key mediator of apoptosis. However, apoptotic neurodegeneration can be prevented by Bcl-2, antiapoptotic protein localizing at the outer mitochondrial membrane (Zhao et al., 2003). Recent findings also demonstrate that polyphenolic compounds in plants can attenuate brain damage in hippocampus following cerebral ischemia via up-regulation of Bcl-2.24 (Li et al., 2012). Based on these pieces of information, we did suggest that $M$. alba powder enhanced the density of 
Bcl-2 immunopositive neuron and decreased caspase-3 immunopositive neuron density in hippocampus resulting in the increased neuron density in the mentioned area. Since apoptosis can occur both via caspase dependent and caspase independent (Zhan et al., 2001), the result demonstraed association between density of caspase-3 immunopositive neuron density and neuron density and association between caspase- 3 and Bcl-2 immunopositive neuron densities in hippocampus were observed. In addition, the neurodegeneration following cerebral ischemia can occur both via apoptosis and necrosis (Rizk et al., 2006) and this is also responsible for the lack of tight association between neuron density and both caspase-3 and Bcl-2 immunopositive neuron densities in hippocampus.

The active phytonutrient of this study is Carica papaya, in general presented in different varieties species which are characterized by their colorant. This plant is a high nutrients efficacy called Srisupa Thai papaya. It is a rich source of beta-carotene. Thus, the current study was set up to further investigate the neuropharmacological effects of Thai Carica papaya. This study demonstrated that the Carica papaya could decreased caspase-3 immunopositive neuron density and the increased Bcl-2immunopositive neurons might be partly responsible for neuroprotective effect of Carica papaya.

\section{Conclusion}

Carica papaya is the potential functional food that can protect against brain damage in apoptotic pathway. The possible underlying mechanisms are associated with the increased survival neurons density via the decreased apoptosis. However, further researches about possible other active ingredient is still necessary.

\section{Acknowledgement}

The financial of this study was supported by the College of research Methodology and Cognitive Science, Burapha University, Thailand

\section{Ethics}

The authors declare no conflicts of interest and states that the research content of this article complies with the ethical standards required by the American Journal of Biochemistry and Biotechnology.

\section{References}

Aruoma, O.I., R. Colognato, I. Fontana, J. Gartlon and L. Migliore et al., 2006. Molecular effects of fermented papaya preparation on oxidative damage, MAP kinase activation and modulation of the benzo[a]pyrene mediated genotoxicity. Biofactors, 26: 147-159. DOI: 10.1002/biof.5520260205
Broughton, B.R., D.C. Reutens and C.G. Sobey, 2009. Apoptotic mechanisms after cerebral ischemia. Stroke, 40: e331-e339.

DOI: $10.1161 /$ STROKEAHA.108.531632

Chen, J., T. Nagayama, K. Jin, R.A. Stetler and R.L. Zhu et al., 1998. Induction of caspase-3-like protease may mediate delayed neuronal death in the hippocampus after transient cerebral ischemia. J. Neurosci., 18: 4914-4928. DOI: 10.1523/JNEUROSCI.18-13-04914.1998

Kaewkaen, P., J. Wattanathorn, T. Thong-Un, S. Muchimapura and P. Wannanond et al., 2012. Mulberry fruits extract mitigate vascular dementia. Am. J. Applied Sci., 9: 1789-1795.

DOI: 10.3844 /ajassp.2012.1789.1795

Kim, G.W., T. Sugawara and P.H. Chan, 2000. Involvement of oxidative stress and caspase- 3 in cortical infarction after photothrombotic ischemia in mice. J. Cereb. Blood Flow Metab., 20: 1690-1701. DOI: 10.1097/00004647-200012000-00008

Koizumi, J., Y. Yoshida, T. Nakazawa and G. Ooneda, 1996. Experimental studies of ischemic brain edema. I. A new experimental model of cerebral embolism in rats in which recirculation can be introduced in the ischemic area. Jpn. Stroke J., 8: 1-8. DOI: $10.3995 /$ jstroke.8.1

Li, Z., L. Pang, F. Fang, G. Zhang and J. Zhang et al., 2012. Resveratrol attenuates brain damage in a rat model of focal cerebral ischemia via up-regulation of hippocampal Bcl-2. Brain Res., 1450: 116-124. DOI: 10.1016/j.brainres.2012.02.019

Lichtenthaler, K. and A.R. Welburn, 1983. Determination of total carotenoids and chlorophylls $\mathrm{A}$ and $\mathrm{B}$ of leaf extracts in different solvents. Biochem. Society Trans., 11: 591-592. DOI: $10.1042 / \mathrm{bst} 0110591$

Liu, R., H. Yuan, F. Yuan and S.H. Yang, 2012. Neuroprotection targeting ischemic penumbra and beyond for the treatment of ischemic stroke. Neurol. Res., 34: 331-337.

DOI: $10.1179 / 1743132812$ Y.0000000020

Longa, E.Z., P.R. Weinstein and S. Carlson, 1989. Reversible middle cerebral artery occlusion without craniectomy in rats. Cummins R Stroke, 20: 84-91. DOI: 10.1161/01.STR.20.1.84

Mehdipour, S., N. Yasa, G. Dehghan, R. Khorasani and A. Mohammadirad et al., 2006. Antioxidant potentials of Iranian Carica papaya juice in vitro and in vivo are comparable to $\alpha$-tocopherol. Phytotherapy Res., 20: 591-594. DOI: $10.1002 /$ ptr. 1932

Ma, L., H.L. Dou, Y.Q. Wu, Y.M. Huang and Y.B. Huang et al., 2012. Lutein and zeaxanthin intake and the risk of age-related macular degeneration: A systematic review and meta-analysis. Br. J. Nutr., 107: 350-359. DOI: 10.1017/S0007114511004260 
Markus, H.S., 2004. Cerebral perfusion and stroke. J. Neurol. Neurosurgery Psychiatry, 75: 353-361. DOI: $10.1136 /$ jnnp.2003.025825

Mergenthaler, P., U. Lindauer, G.A. Dienel and A. Meisel, 2013. Sugar for the brain: the role of glucose in physiological and pathological brain function. Trends Neurosci., 36: 587-597. DOI: 10.1016/j.tins.2013.07.001

Palace, V.P., N. Khaper, Q. Qin and P.K. Singal, 1999. Anti-oxidant potentials of vitamin A and carotenoids and their relevance to heart disease. Free Rad Biol Med, 26: 746-61. DOI: $10.1016 / \mathrm{S} 0891-5849(98) 00266-4$

Pendzhiev, A.M., 2002. Proteolytic enzymes of papaya: Medicinal applications. Pharmaceutical Chem. J., 36: 315-317. DOI: $10.1023 / A: 1020832807958$

Raza, S.S., M.M. Khan, M. Ashafaq, A. Ahmad and G. Khuwaja et al., 2011. Silymarin protects neurons from oxidative stress associated damages in focal cerebral ischemia: A behavioral, biochemical and immunohistological study in Wistar rats. J. Neurol. Sci., 309: 45-54. DOI: $10.1016 /$ j.jns.2011.07.035

Rainha, N., E. Lima, J. Baptista and C. Rodrigues, 2011. Antioxidant properties, total phenolic, total carotenoid and chlorophyll content of anatomical parts of Hypericum foliosum. J. Medicinal Plants Res., 5: 1930-1940.

Ren, M., Z.J. Lin, H. Qian, C.R. Gourav and R. Liu et al., 2012. Embolic middle cerebral artery occlusion model using thrombin and fibrinogen composed clots in rat. J. Neurosci. Meth., 211: 296-304. DOI: $10.1016 /$ j.jneumeth.2012.09.006

Rizk, N.N., J.A. Rafols and J.C. Dunbar, 2006. Cerebral ischemia-induced apoptosis and necrosis in normal and diabetic rats: Effects of insulin and C-peptide. Brain Res., 1096: 204-212. DOI: $10.1016 /$ j.brainres.2006.04.060

Rojsanga, P., P. Sithisarn and S. Buranaphalin, 2014. Validated UV spectrophotometric method for quantitative analysis of carotenoid content and antioxidant activities of Pluk Mai Lie Papaya Fruits. Pharm. Sci. Asia, 41: 41-47.
Sacco, R.L., S.E. Kasner, J.P. Broderick, L.R. Caplan and J.J. Connors et al., 2013. American heart association stroke council, council on cardiovascular surgery and anesthesia; council on cardiovascular radiology and intervention; council on cardiovascular and stroke nursing; council on epidemiology and prevention; council on peripheral vascular disease; council on nutrition, physical activity and metabolism. An updated definition of stroke for the 21st century: A statement for healthcare professionals from the American Heart Association/American Stroke Association. Stroke, 44: 2064-2089.

DOI: 10.1161/STR.0b013e318296aeca

Slater, A.F., C.S. Nobel and S. Orrenius, 1995. The role of intracellular oxidants in apoptosis. Biochim. Biophys. Acta, 1271: 59-62.

DOI: 10.1016/0925-4439(95)00010-2

Seki, T., I. Yajima, T. Yabu, M. Ooguri and J. Nakanishi et al., 2007. Examining an Exfoliation Promoting Enzyme for Cosmetic Applications. In: Biotechnology in Cosmetics: Concepts, Tools and Techniques, Koslowski, A. (Ed.), Allured Pub. Co., Illinois, ISBN-10: 1932633243, pp: 285-300.

Wang-Fischer, Y., 2009. Manual of Stroke Models in Rat. 1st Edn., CRC Press, Boca Raton (FL), ISBN-10: 0203881087, pp: 352.

Zhan, R.Z., C. Wu, H. Fujihara, K. Taga and S. Qi et al., 2001. Both caspase-dependent and caspaseindependent pathways may be involved in hippocampal CA1 neuronal death because of loss of cytochrome c From mitochondria in a rat forebrain ischemia model. J. Cereb Blood Flow Metab., 21: 529-540.

DOI: 10.1097/00004647-200105000-00007

Zhao, H., M.A. Yenari, D. Cheng, R.M. Sapolsky and G.K. Steinberg, 2003. Bcl-2 overexpression protects against neuron loss within the ischemic margin following experimental stroke and inhibits cytochrome c translocation and caspase-3 activity. J. Neurochem., 85: 1026-1036. DOI: $10.1046 /$ j.1471-4159.2003.01756.x 